\title{
The chromosomal distribution of sex-biased microRNAs in Drosophila is non-adaptive
}

\author{
Antonio Marco \\ School of Life Sciences, University of Essex, Colchester, United Kingdom.
}

Short title: Why is the $\mathrm{X}$ chromosome rich in male-expressed microRNAs?

Correspondence should be addressed to:

Antonio Marco

School of Life Sciences

University of Essex, Wivenhoe Park, Colchester CO4 3SQ, United Kingdom

Email: amarco.bio@gmail.com

Telephone: +44 (0) 1206873339 


\section{ABSTRACT}

Genes are often differentially expressed between males and females. In Drosophila melanogaster, the analysis of sex-biased microRNAs (short non-coding regulatory molecules) has revealed striking differences with protein-coding genes. Mainly, the X chromosome is enriched in male-biased microRNA genes, although it is depleted of male-biased protein-coding genes. The paucity of male-biased genes in the X chromosome is generally explained by an evolutionary process called demasculinization. I suggest that the excess of male-biased microRNAs in the X chromosome is due to high-rates of de novo emergence of microRNAs, a tendency of novel microRNAs in the $\mathrm{X}$ chromosome to be expressed in testis, and to a lack of a demasculinization process. To test this hypothesis I analysed the expression profile of microRNAs in males, females and gonads in D. pseudoobscura, in which an autosome translocated into the $\mathrm{X}$ chromosome effectively becoming part of a sex chromosome (neo-X). I found that the pattern of sex- biased expression is generally conserved between D. melanogaster and D. pseudoobscura. Also, orthologous microRNAs in both species conserve their chromosomal location, indicating that there is no evidence of demasculinization or other inter-chromosomal movement of microRNAs. D. pseudoobscura-specific microRNAs in the neo-X chromosome tend to be male-biased and particularly expressed in testis. In summary, the apparent paradox resulting from male-biased protein-coding genes depleted in the $\mathrm{X}$ chromosome and an enrichment in male-biased microRNAs is a consequence of different evolutionary dynamics between coding genes and short RNAs. 


\section{INTRODUCTION}

Gene expression is tightly regulated by mechanisms that make gene products to be expressed in specific organs at specific times. This spatiotemporal control of gene expression determines the development of fertilised eggs into adult organisms (Wolpert et al. 2006; Davidson 2006). In organisms with two sexes a large fraction of genes are also differentially expressed between males and females (Parisi et al. 2003; Ranz et al. 2003). Also, most male or female specific expression occurs in the gonads, alhough other tissues also show sex-expression bias (Parisi et al. 2004; Chang et al. 2011). Female biased genes tend to be located in the X chromosome (in XY systems) although male-biased genes tend to be depleted in the X chromosome (Parisi et al. 2003; Ranz et al. 2003; Khil et al. 2004).

In the model species Drosophila melanogaster the study of sex-biased expression indicates that male-biased genes often appear in the X chromosome (Arguello et al. 2006; Levine et al. 2006; Begun et al. 2007; Chen et al. 2007), but they are later retroposed (copied) to an autosome and the original copy is eventually lost (Zhang et al. 2010b). This process, called 'demasculinization', was predicted already by early theoretical models (Rice 1984), and recent works seems to support it (Jiang and Machado 2009; Bachtrog et al. 2010; Zhang et al. 2010a; b), although there is still some controversy around it alternative models should be taken into consideration (Meiklejohn and Presgraves 2012). However, these conclusions cannot be extrapolated to all gene products, as most work on sex-biased expression has been focused on a specific type of gene: protein-coding genes. Characterising the distinct evolutionary dynamics of 
different types of genes is paramount in evolutionary biology, as it can reveal how genetic and genomic features influence, and ultimately determine, the fate of genes (Lynch 2007).

MicroRNAs, short RNA post-transcriptional regulators [reviewed in (Bartel 2004; Axtell et al. 2011; Marco et al. 2013a)], are also expressed differently in males and in females (Marco et al. 2013b; Marco 2014, 2015). Although an early investigation suggested that they were also subject to demasculinization (Zhang et al. 2010b), a more recent work indicates that this is probably not the case (Marco 2014). Indeed, male microRNAs tend to be enriched in the X chromosome (Mishima et al. 2008; Li et al. 2010), contrary to what is observed in protein-coding genes. In $D$. melanogaster novel microRNAs in the X chromosome are often expressed in testis and most are evolutionarily young (Marco 2014). To investigate the evolutionary dynamics of microRNAs in the X chromosome I characterised the sex-biased microRNA complement of the species Drosophila pseudoobcura, which diverged from D. melanogaster about $25 \mathrm{Myr}$ ago, and had an autosome translocated into an X-chromosome, becoming a recently evolved X-chromosome (or neo-X).

\section{MATERIAL AND METHODS}

Sample collection: The work was done with a Drosophila pseudoobscura stock kindly donated by Tom Price (University of Liverpool) and Nina Wedell (University of Exeter), collected in Show Low (Arizona, USA). All flies were kept at $18^{\circ} \mathrm{C}$ on cornmeal based media, with 12 hours light/dark cycles. Adult males and females were collected at age 7 days. Fertilized eggs were 
collected from virgin females in population cages (about 30-50 females) in cycles of 12 hours. Virgin females were allowed to lay eggs in control vials to ensure they were virgins. We discarded population cages with females coming from control vials having larvae within two weeks (in these experiments, it only happened once). Eggs were collected with a sieve and washed with wash solution $(\mathrm{NaCl} 100 \mathrm{mM}$, Triton $\mathrm{X}-1000.7 \mathrm{mM})$ and water.

RNA extraction and sequencing: Total RNA from samples was extracted using TRIzol (Life Technologies) as recommended by the manufacturer. RNA was dissolved in RNase-free water. For small RNA sequencing I used the TruSeq Small RNA Sample Preparation Kit (Illumina) to generate the cDNA library using selected constructs of sizes 145 to $160 \mathrm{bp}$ in a $6 \%$ PAGE gel, and precipitated in ethanol. DNA integrity was checked with TapeStation (Agilent). Samples were sequenced in-house with an Illumina MiSeq sequencing machine.

Data analysis: Reads were mapped to the Drosophila pseudoobscura genome sequence assembly 104 (Liao et al. 2021) with HISAT2 version 2.2.1 (Kim et al. 2019) using default parameters. Raw reads were deposited in Gene Expression Omnibus (GEO) at NCBI with accession number GSE179989. Additionally, reads from other D. pseudoobscura samples were retrieved from GEO: GSE98013 (Mohammed et al. 2018) (two males and one female samples) and GSE48017 (Lyu et al. 2014) (one ovary and one testis samples) and also mapped to the same reference genome. Reads from D. melanogaster SRR016854, SRR018039, SRR069836 and SRR069837 (Chung et al. 2008; Roy et al. 2010) were mapped with the same method to the reference genome dm6 (Hoskins et al. 2015). Adapters were trimmed with Cutadapt 1.18 (Martin 
2011) prior to mapping. Read counts were obtained with featureCounts 2.0.2 (Liao et al. 2014)

using the annotation coordinates from miRBase release 21 (Kozomara et al. 2018) and the additional microRNAs annotated by (Mohammed et al. 2018), and microRNAs not supported by this study were also removed from the analysis. Differential gene expression was conducted with DESeq2 version 1.24.0 (Love et al. 2014 p. 2) with local fitting, using the model expression batch + sex, where batch is the sequencing run. All data generated and scripts to reproduce the full analysis is available from GitHub: https://github.com/antoniomarco/miRpseudoobscura

\section{RESULTS}

In order to characterize differentially expressed microRNAs between males and females in $D$.

pseudoobscura I generated and sequenced small RNA libraries from males and young unfertilised females (see Methods). There are available small RNA datasets for males and females of this species (Mohammed et al. 2018). However, these datasets have either no biological replicates, or they have been sequencing using different library construction methods. Hence, I sequenced male and female samples in two paired sequencing reactions (one male sample and one female sample in each sequencing array) using the same protocols. The differential gene expression analysis was performed taking into account batch (paired) effects. In any case, the $\log 2$ fold-changes computed for both sets of experiments (ours and the already available) were similar, and a linear model to predict $\log 2$ fold-changes in the external dataset from our values fitted very well ( $\mathrm{p}<0.001 ; \mathrm{R}^{2}=0.35$; Supplementary Information). 
The analysis identified 21 microRNAs with a clear sex-biased expression profile at a False Discovery Rate (FDR) of 10\%, 10 overexpressed in females and 11 in males (Figure 1A). I then reanalysed our previously generated $D$. melanogaster small RNA libraries using the method here described. There were 100 orthologous microRNAs conserved between $D$. melanogaster and $D$. pseudoobscura. The chromosome in which the microRNA is located (Muller element) is conserved for all studied sequences, although some evidence of translocations and inversion are observed within chromosomes (Figure 2). Importantly, there is not a single case of a microRNA in one of the Drosophila species that moved to a different chromosome in the other species. In other word, the analysis did not identify a single microRNA that moved out of a sex chromosome to an autosome (or vice versa), a phenomena that is well described for protein-coding genes, and it generally due to retroposition (see Discussion).

To investigate whether the expression profile of microRNAs change in a different chromosomal context, I compared the sex-bias in expression of orthologogous microRNAs between both Drosophila species. A scatter plot of the $\log 2$ fold-change values for pairs of orthologs reveals that the expression bias is conserved between both species (Figure 1B). The female/male expression bias is also conserved in microRNAs that are autosomal in D. melanogaster but that are in the new-X chromosome in D. pseudoobscura (Figure 1B; fitted linear model $\mathrm{p}=0.007$ $\mathrm{R}^{2}=0.3$ ), showing evidence that the chromosomal context (sex versus autosomal) does not change the sex-biased expression pattern. 
To evaluate the expression-bias of novel and conserved microRNAs depending on the chromosomal context, I compared the expression bias ( $\log 2$ fold-change) of novel and conserved microRNAs in both autosomes and the X chromosome in D. pseudoobscura. Novel microRNAs that emerged in the $\mathrm{X}$ chromosome tended to be male expressed compared to the other microRNAs, although the difference was small (Figure 1C). However, when I compare the testis to ovary expression ratio for microRNAs expressed in the gonads, it is clear that novel microRNAs in the $\mathrm{X}$ tend to be overexpressed in testis (Figure 1D; $\mathrm{p}=0.00018$ Mann-Whitney test).

\section{DISCUSSION}

In this work I characterised the sex expression patter of microRNAs in Drosophila pseudoobscura to investigate the evolutionary dynamics of male expressed microRNAs. By comparing the expression profile of $D$. pseudoobscura and D. melanogaster it was evident that the sex-bias of microRNAs is largely conserved between both species. This is also the case for microRNAs that were located in an autosome which became the neo-X arm after a translocation, supporting that the relative expression between males and females does not depend on the chromosomal context. In other words, male-biased microRNAs may acquire their expression profile early during evolution, but then their expression is maintained even if the chromosome acquires a sex chromosome status. The conservation of the expression bias as well as the conservation of the chromosome in which microRNAs are located provides strong evidence against demasculinization having any impact in microRNAs. 
The demasculinization model described in the Introduction assumes that male-expressed (male-benefitial) genes are more likely to be evolutionarily lost if they are located in the $\mathrm{X}$ chromosome, as damaging mutations are often lethal in hemizygosis (Figure 3A). Hence, gene copies in the autosome are favoured while the ancestral X located copy is eventually lost (Sturgill et al. 2007). The genes are copied by retroposition (Figure 3B), in which a reverse transcriptase makes a DNA copy of the processed transcript which is inserted (by a retroviral integrase) in the genome. The original copies of genes retroposed into the autosomes are eventually lost (Figure 3C). This mechanism is highly unlikely, if not impossible, in microRNAs since processed microRNA precursors leaving the nucleus (and therefore exposed to endogenous reverse transcriptases) are short (less than 100 nucleotides) RNA molecules with an internal hairpin structure (Bartel 2004), lacking a polyadenylation sequence that could be used as a primer by the endogenous reverse transcriptases (Wei et al. 2001).

This work supports an alternative model of evolution. MicroRNAs have a high rate of turn-over. That is, novel microRNAs appear at high frequencies (Figure 3D), which is less common in protein-coding genes. Novel genes are often male-biased (Metta and Schlötterer 2008), and if they are in the $\mathrm{X}$ they are frequently highly expressed in testis (this work). This bias in gene expression of newly emerged microRNAs, together with the high rate of evolutionarily young microRNA loss (Figure 3E) eventually leads to an enrichment of male (testis) expressed microRNAs in the $\mathrm{X}$ chromosome (Figure 3F).

It is also important to keep into consideration that male-biased expression does not necessarily mean beneficial for males, or that losing the gene males fertility will be compromised. Indeed, 
genes whose lack of function lead to infertility are male expressed, but the converse does not hold true, male-biased genes are not generally fertility genes (Lindsley et al. 2013). Putting all this together, this work strongly suggest that the X-chromosome is enriched in male-biased microRNAs as a consequence of mutation bias (tendency of novel microRNAs in the $\mathrm{X}$ to be male biased), lack of retroposition, and a conservation of the expression-bias independently of the chromosomal context. In summary, the evidence supports that the chromosomal location of sex-biased microRNAs is non-adaptive.

\section{ACKNOWLEDGEMENTS}

I would like to thank Tom Price and Nina Wedell for sharing their Drosophila pseudoobscura stocks. I am also grateful to J. J. Emerson for helping me to understand the chromosome assembly of the most recent $D$. pseudoobscura genome assembly. This work was supported by the University of Essex.

\section{REFERENCES}

Arguello J. R., Y. Chen, S. Yang, W. Wang, and M. Long, 2006 Origination of an X-Linked Testes Chimeric Gene by Illegitimate Recombination in Drosophila. PLoS Genet 2: e77. https://doi.org/10.1371/journal.pgen.0020077

Axtell M. J., J. O. Westholm, and E. C. Lai, 2011 Vive la différence: biogenesis and evolution of microRNAs in plants and animals. Genome Biol. 12: 221. https://doi.org/10.1186/gb-2011-12-4-221

Bachtrog D., N. R. T. Toda, and S. Lockton, 2010 Dosage Compensation and Demasculinization of X Chromosomes in Drosophila. Curr. Biol. 20: 1476-1481. https://doi.org/10.1016/j.cub.2010.06.076 
Bartel D. P., 2004 MicroRNAs: genomics, biogenesis, mechanism, and function. Cell 116: 281-297.

Begun D. J., H. A. Lindfors, A. D. Kern, and C. D. Jones, 2007 Evidence for de Novo Evolution of Testis-Expressed Genes in the Drosophila yakuba/Drosophila erecta Clade. Genetics 176: 1131-1137. https://doi.org/10.1534/genetics.106.069245

Chang P. L., J. P. Dunham, S. V. Nuzhdin, and M. N. Arbeitman, 2011 Somatic sex-specific transcriptome differences in Drosophila revealed by whole transcriptome sequencing. BMC Genomics 12: 364. https://doi.org/10.1186/1471-2164-12-364

Chen S.-T., H.-C. Cheng, D. A. Barbash, and H.-P. Yang, 2007 Evolution of hydra, a recently evolved testis-expressed gene with nine alternative first exons in Drosophila melanogaster. PLoS Genet. 3: e107. https://doi.org/10.1371/journal.pgen.0030107

Chung W.-J., K. Okamura, R. Martin, and E. C. Lai, 2008 Endogenous RNA interference provides a somatic defense against Drosophila transposons. Curr. Biol. CB 18: 795-802. https://doi.org/10.1016/j.cub.2008.05.006

Hoskins R. A., J. W. Carlson, K. H. Wan, S. Park, I. Mendez, et al., 2015 The Release 6 reference sequence of the Drosophila melanogaster genome. Genome Res. 25: 445-458. https://doi.org/10.1101/gr.185579.114

Jiang Z.-F., and C. A. Machado, 2009 Evolution of Sex-Dependent Gene Expression in Three Recently Diverged Species of Drosophila. Genetics 183: 1175-1185. https://doi.org/10.1534/genetics.109.105775

Khil P. P., N. A. Smirnova, P. J. Romanienko, and R. D. Camerini-Otero, 2004 The mouse X chromosome is enriched for sex-biased genes not subject to selection by meiotic sex chromosome inactivation. Nat. Genet. 36: 642-646. https://doi.org/10.1038/ng1368

Kim D., J. M. Paggi, C. Park, C. Bennett, and S. L. Salzberg, 2019 Graph-based genome alignment and genotyping with HISAT2 and HISAT-genotype. Nat. Biotechnol. 37: 907-915. https://doi.org/10.1038/s41587-019-0201-4

Kozomara A., M. Birgaoanu, and S. Griffiths-Jones, 2018 miRBase: from microRNA sequences to 
function. Nucleic Acids Res. https://doi.org/10.1093/nar/gky1141

Levine M. T., C. D. Jones, A. D. Kern, H. A. Lindfors, and D. J. Begun, 2006 Novel Genes Derived from Noncoding DNA in Drosophila Melanogaster Are Frequently X-Linked and Exhibit Testis-Biased Expression. Proc. Natl. Acad. Sci. 103: 9935-9939. https://doi.org/10.1073/pnas.0509809103

Li J., Y. Liu, D. Dong, and Z. Zhang, 2010 Evolution of an X-Linked Primate-Specific Micro RNA Cluster. Mol. Biol. Evol. 27: 671-683. https://doi.org/10.1093/molbev/msp284

Liao Y., G. K. Smyth, and W. Shi, 2014 featureCounts: an efficient general purpose program for assigning sequence reads to genomic features. Bioinforma. Oxf. Engl. 30: 923-930. https://doi.org/10.1093/bioinformatics/btt656

Liao Y., X. Zhang, M. Chakraborty, and J. J. Emerson, 2021 Topologically associating domains and their role in the evolution of genome structure and function in Drosophila. Genome Res. 31: 397-410. https://doi.org/10.1101/gr.266130.120

Lindsley D. L., J. Roote, and J. A. Kennison, 2013 Anent the Genomics of Spermatogenesis in Drosophila melanogaster. PLoS ONE 8: e55915. https://doi.org/10.1371/journal.pone.0055915

Love M. I., W. Huber, and S. Anders, 2014 Moderated estimation of fold change and dispersion for RNA-seq data with DESeq2. Genome Biol. 15: 550. https://doi.org/10.1186/s13059-014-0550-8

Lynch M., 2007 The Origins of Genome Architecture. Sinauer Associates Inc, Sunderland.

Lyu Y., Y. Shen, H. Li, Y. Chen, L. Guo, et al., 2014 New MicroRNAs in Drosophila—Birth, Death and Cycles of Adaptive Evolution. PLoS Genet 10: e1004096. https://doi.org/10.1371/journal.pgen.1004096

Marco A., M. Ninova, and S. Griffiths-Jones, 2013a Multiple products from microRNA transcripts. Biochem. Soc. Trans. 41: 850-854. https://doi.org/10.1042/BST20130035

Marco A., A. Kozomara, J. H. L. Hui, A. M. Emery, D. Rollinson, et al., 2013b Sex-Biased Expression of MicroRNAs in Schistosoma mansoni. PLoS Negl Trop Dis 7: e2402. https://doi.org/10.1371/journal.pntd.0002402

Marco A., 2014 Sex-biased expression of microRNAs in Drosophila melanogaster. Open Biol. 4: 140024. 
https://doi.org/10.1098/rsob.140024

Marco A., 2015 Selection Against Maternal microRNA Target Sites in Maternal Transcripts. G3 GenesGenomesGenetics 5: 2199-2207. https://doi.org/10.1534/g3.115.019497

Martin M., 2011 Cutadapt removes adapter sequences from high-throughput sequencing reads. EMBnet.journal 17: 10-12. https://doi.org/10.14806/ej.17.1.200

Meiklejohn C. D., and D. C. Presgraves, 2012 Little evidence for demasculinization of the Drosophila X chromosome among genes expressed in the male germline. Genome Biol. Evol. 4: 1007-1016. https://doi.org/10.1093/gbe/evs077

Metta M., and C. Schlötterer, 2008 Male-biased genes are overrepresented among novel Drosophila pseudoobscura sex-biased genes. BMC Evol. Biol. 8: 182. https://doi.org/10.1186/1471-2148-8-182

Mishima T., T. Takizawa, S.-S. Luo, O. Ishibashi, Y. Kawahigashi, et al., 2008 MicroRNA (miRNA) cloning analysis reveals sex differences in miRNA expression profiles between adult mouse testis and ovary. Reproduction 136: 811-822. https://doi.org/10.1530/REP-08-0349

Mohammed J., A. S. Flynt, A. M. Panzarino, M. M. H. Mondal, M. DeCruz, et al., 2018 Deep experimental profiling of microRNA diversity, deployment, and evolution across the Drosophila genus. Genome Res. 28: 52-65. https://doi.org/10.1101/gr.226068.117

Parisi M., R. Nuttall, D. Naiman, G. Bouffard, J. Malley, et al., 2003 Paucity of Genes on the Drosophila X Chromosome Showing Male-Biased Expression. Science 299: 697-700. https://doi.org/10.1126/science. 1079190

Parisi M., R. Nuttall, P. Edwards, J. Minor, D. Naiman, et al., 2004 A survey of ovary-, testis-, and soma-biased gene expression in Drosophila melanogaster adults. Genome Biol. 5: R40. https://doi.org/10.1186/gb-2004-5-6-r40

Ranz J. M., C. I. Castillo-Davis, C. D. Meiklejohn, and D. L. Hartl, 2003 Sex-Dependent Gene Expression and Evolution of the Drosophila Transcriptome. Science 300: 1742-1745. https://doi.org/10.1126/science.1085881 
Rice W. R., 1984 Sex Chromosomes and the Evolution of Sexual Dimorphism. Evolution 38: 735-742. https://doi.org/10.1111/j.1558-5646.1984.tb00346.x

Roy S., J. Ernst, P. V. Kharchenko, P. Kheradpour, N. Negre, et al., 2010 Identification of functional elements and regulatory circuits by Drosophila modENCODE. Science 330: 1787-1797. https://doi.org/10.1126/science. 1198374

Sturgill D., Y. Zhang, M. Parisi, and B. Oliver, 2007 Demasculinization of X chromosomes in the Drosophila genus. Nature 450: 238-241. https://doi.org/10.1038/nature06330

Wei W., N. Gilbert, S. L. Ooi, J. F. Lawler, E. M. Ostertag, et al., 2001 Human L1 retrotransposition: cis preference versus trans complementation. Mol. Cell. Biol. 21: 1429-1439. https://doi.org/10.1128/MCB.21.4.1429-1439.2001

Zhang Y. E., M. D. Vibranovski, P. Landback, G. A. B. Marais, and M. Long, 2010a Chromosomal Redistribution of Male-Biased Genes in Mammalian Evolution with Two Bursts of Gene Gain on the X Chromosome. PLoS Biol 8: e1000494. https://doi.org/10.1371/journal.pbio.1000494

Zhang Y. E., M. D. Vibranovski, B. H. Krinsky, and M. Long, 2010b Age-dependent chromosomal distribution of male-biased genes in Drosophila. Genome Res. 20: 1526-1533. https://doi.org/10.1101/gr.107334.110 


\section{FIGURE LEGENDS}

Figure 1. Sex-biased expression of microRNAs in Drosophila pseudoobscura. (A) Smear plot of expressed microRNAs. Black dots are differentially expressed genes between males and females. The $\log 2$ fold-change in the Y-axis is positive for male-biased genes and negative for female-biased genes. (B) Scatter-plot of $\log 2$ fold-change expression values of D. melanogaster and D. pseudoobscura. Black dots represent microRNAs located in the neo-X chromosome in $D$. pseudoobscura, and the other microRNAs are in grey, and black and grey straight lines represent the linear model fitted to both groups respectively. Dashed line is the 1:1 ratio. (C) Log2 fold-change of male versus female expression for conserved or D. pseudoobscura specific (novel) microRNAs, either autosomal (A) or in the X chromosome (X). (D) Log2 fold-change of testis versus ovary expression.

Figure 2. Comparison of the chromosomal location of microRNAs between $D$. melanogaster and D. pseudoobscura. Homologous chromosomes of both species are paired, and grey lines connect the location of orthologous microRNAs.

Figure 3. Models of protein-coding and microRNA genes evolution. (A) Effect of deleterious mutations in male genes. (B) Retroposition out of the X chromosome. (C) Deletion of the original copy of a retroposed gene. (D) Emergence of novel microRNAs in the X chromosome highly-expressed in testis (in blue) and in autosomes. (E) Random deleterious mutations in microRNA genes. (F) Enrichment of male-biased microRNAs in the X chromosome. Details of the models are in the main text. 
bioRxiv preprint doi: https://doi.org/10.1101/2021.09.25.461796; this version posted September 25, 2021. The copyright holder for this preprint (which was not certified by peer review) is the author/funder, who has granted bioRxiv a license to display the preprint in perpetuity. It is made available under aCC-BY 4.0 International license.

\section{Figure 1}

(A)

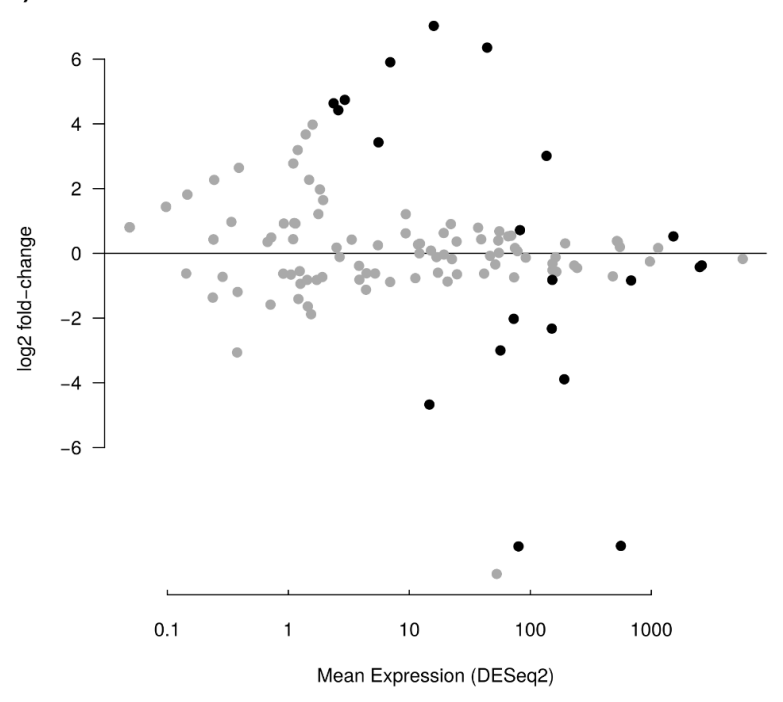

(C)

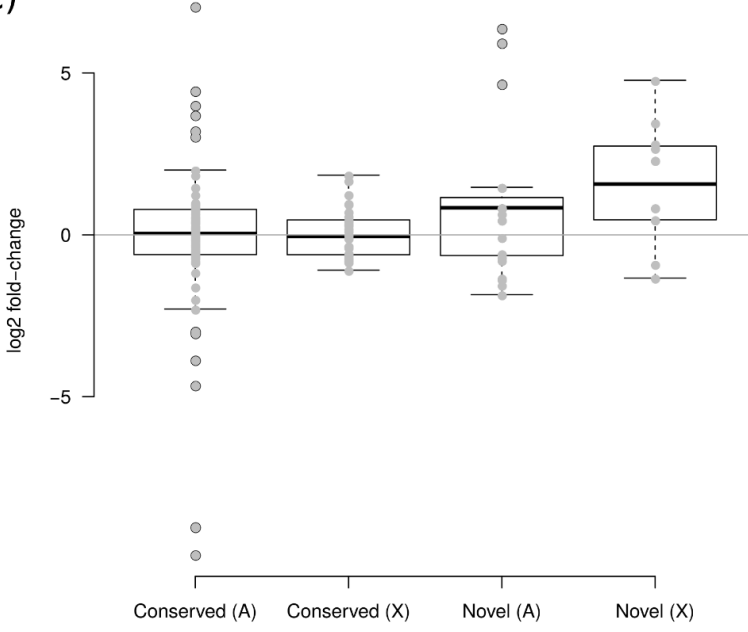

(B)

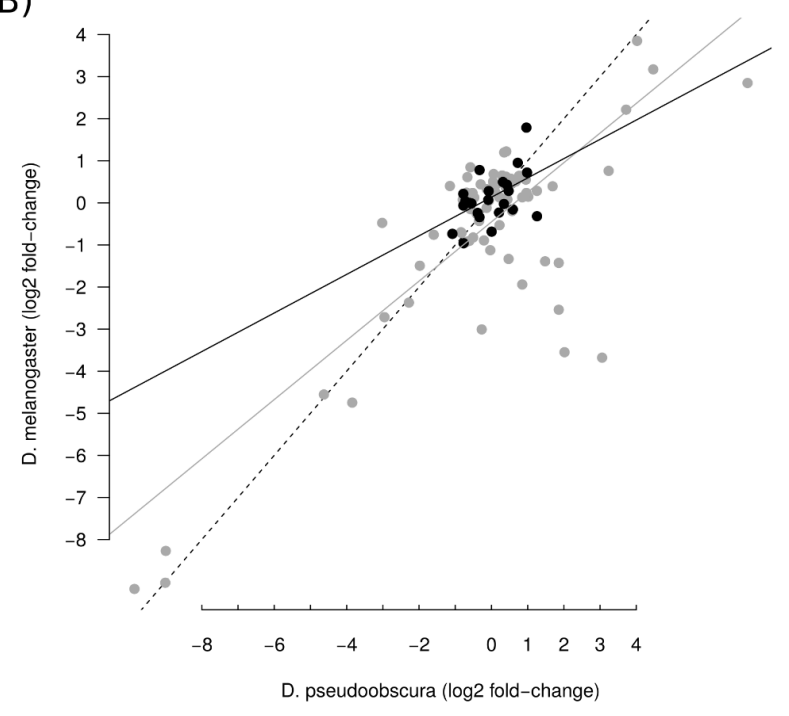

(D)

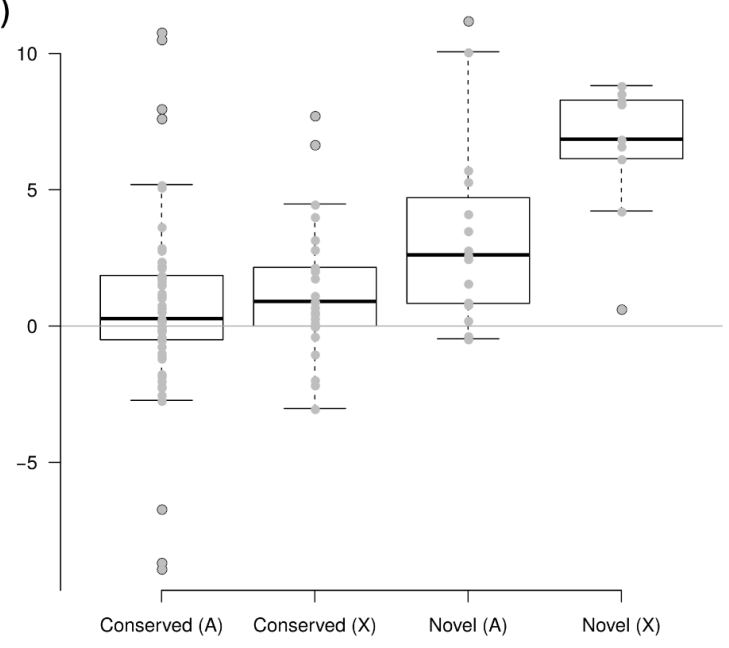


bioRxiv preprint doi: $h$ ttps://doi.org/10.1101/2021.09.25.461796; this version posted September 25, 2021. The copyright holder for this preprint (which was not certified by peer review) is the author/funder, who has granted bioRxiv a license to display the preprint in perpetuity. It is made available under aCC-BY 4.0 International license.

\section{Figure 2}
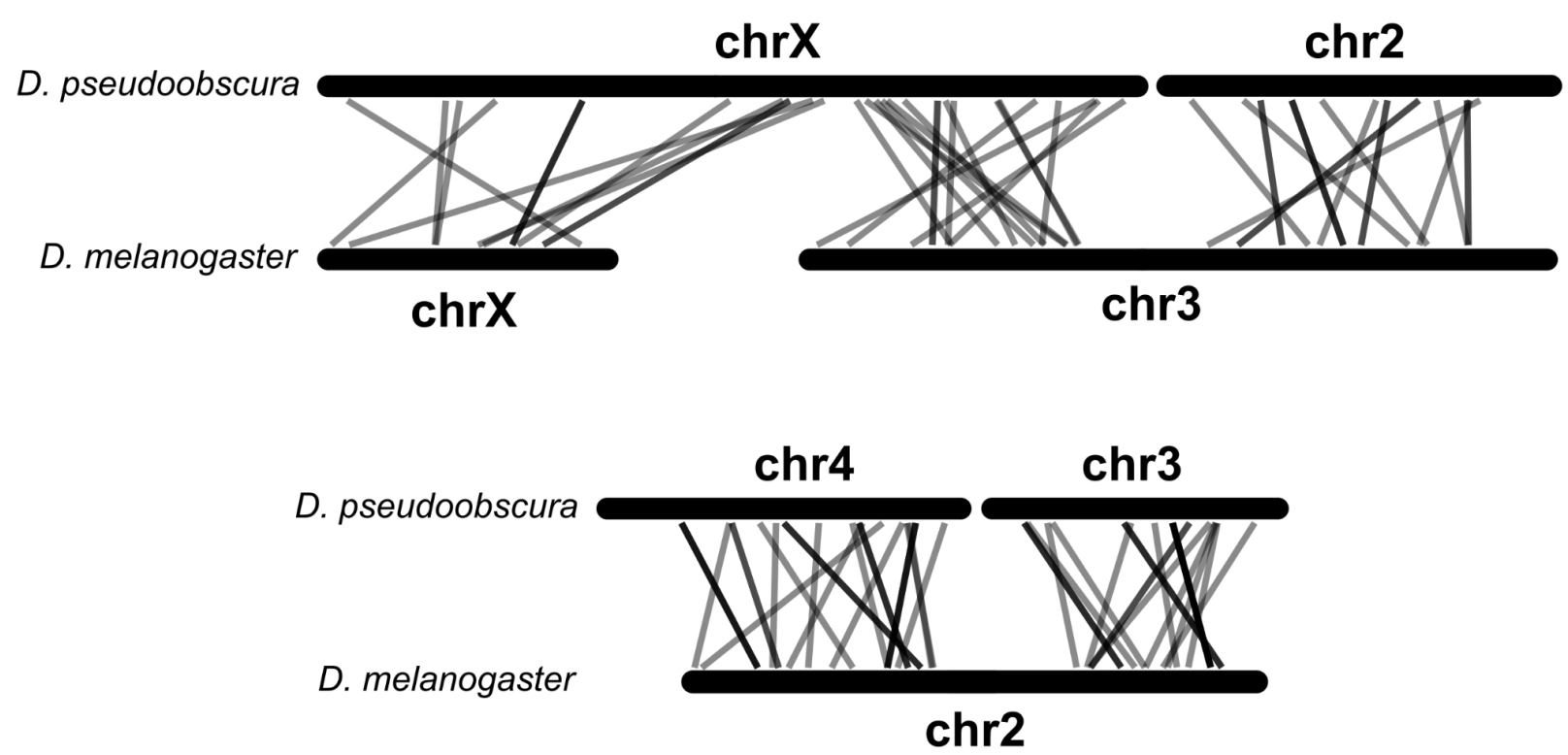
bioRxiv preprint doi: https://doi.org/10.1101/2021.09.25.461796; this version posted September 25, 2021. The copyright holder for this preprint (which was not certified by peer review) is the author/funder, who has granted bioRxiv a license to display the preprint in perpetuity. It is made available under aCC-BY 4.0 International license.

\section{Figure 3}

(A)

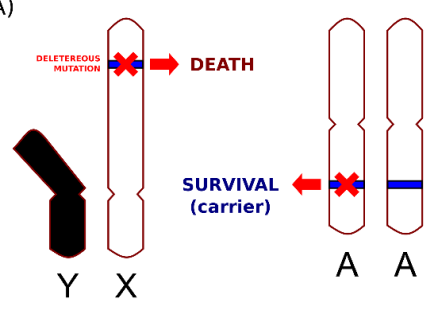

(D)

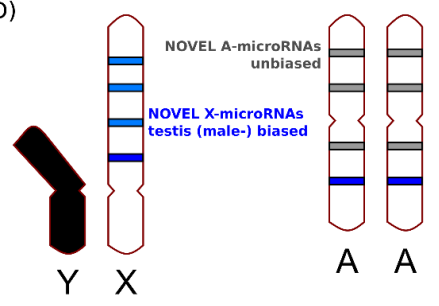

(B)

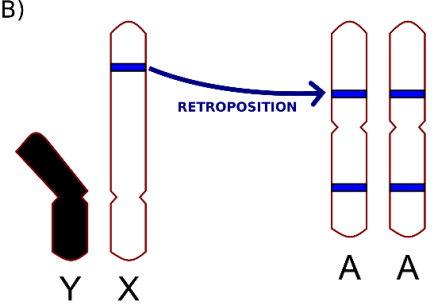

(E)
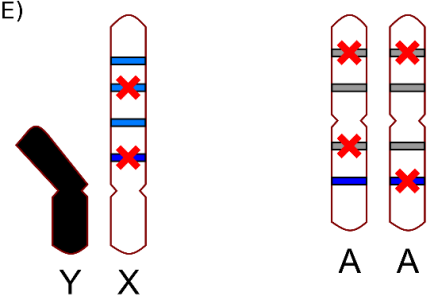

A A
(C)
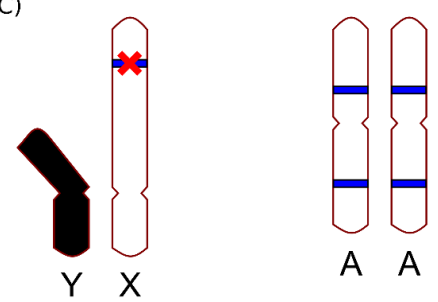

A A

(F)

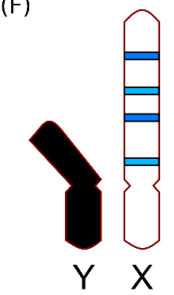

A $A$ 


\section{Supplementary Figure 1}

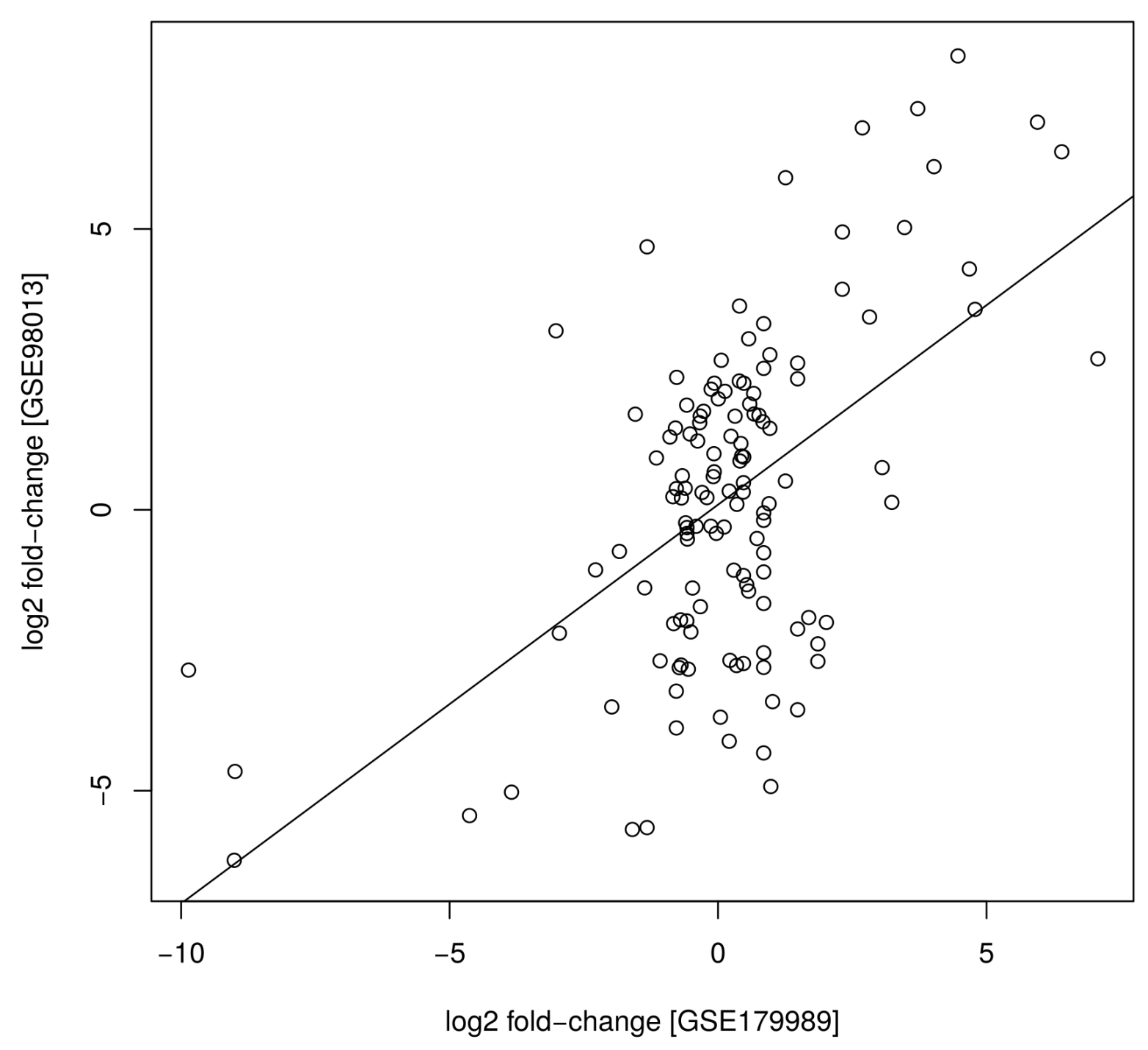

Supplementary Figure 1. Log2 fold-change values estimated with DESeq2 for male versus female expression levels, for the dataset here described (GSE179989) and a previous set of sequencing experiments (GSE98013). 